\title{
Bench testing of auto-adjusting positive airway pressure devices
}

\author{
F. Abdenbi, B. Chambille, P. Escourrou
}

Bench testing of auto-adjusting positive airway pressure devices. F. Abdenbi, B. Chambille, P. Escourrou. (C) ERS Journals Ltd 2004.

ABSTRACT: Continuous positive airway pressure devices are routinely used to treat sleep breathing disorders. Automated devices that adjust the therapeutic pressure have recently been proposed. The utility of such devices is still controversial, as rigorous clinical comparisons are difficult to perform as a result of patient and device differences.

The current authors studied automated devices in a respiratory model that was able to mimic upper airway mechanics and to interact with pressure adjustment in a closed loop. Five auto-adjusted devices were submitted to this model, in order to determine their ability to detect respiratory events and adjust pressure accordingly.

All apnoeas were suppressed, whilst the reaction to repetitive hypopnoeas was dependent on the airflow shape. In some devices, repetitive hypopnoeas were changed to flow limitation. Artificial snoring caused a pressure increase in four devices, and constant mask leak was not systematically compensated. Only one device did not raise pressure in response to central apnoeas with opened upper airways. These findings show that, in some devices, event classification failed and normal airflow was not fully restored, resulting in elevated residual event indices.

In conclusion, this model is useful in order to reproducibly compare diagnostic and therapeutic capacities of commercial devices as a first step, before costly clinical studies.

Eur Respir J 2004; 24: 649-658.
Explorations Fonctionnelles Multidisciplinaires, Hôpital Antoine Béclère, Clamart, France.

Correspondence: F. Abdenbi, Explorations Fonctionnelles Multidisciplinaires, Hôpital Antoine Béclère, 157 rue de la porte de Trivaux, 92140 Clamart, France.

Fax: 33145374828

E-mail: faycal.abdenbi@kb.u-psud.fr

Keywords: Automation

flow limitation

sleep apnoea syndromes

snoring

upper airway mechanics

Received: December 32003

Accepted after revision: June 162004

This work was supported by Réseau National des Technologies pour la Santé (RNTS) convention no. 014906028.
Patients with obstructive sleep apnoea syndrome (OSAS) are often chronically treated with continuous positive airway pressure (CPAP), which may induce several side-effects, limiting treatment tolerance and compliance. Auto-adjusting positive airway pressure (APAP) systems have been developed and clinically tested for several years [1-7]. They are aimed at improving effectiveness and, possibly, reducing side-effects of CPAP by providing optimal pressure, which is found, on average, to be lower than a continuous level [8]. They may improve tolerance by reducing mask pressure $(P \mathrm{~m})$ and, probably, mask leaks. It has been demonstrated that, in patients requiring high pressures, compliance was statistically increased [7]. However, the clinical significance of this observation remains unproven. APAP are also potentially cost-efficient auto-titrating systems $[9,10]$. Nevertheless, in some reports, failure of the APAP required manual resetting, whilst, in others, undesirable cardiorespiratory complications have been described [11-14].

The principle of these machines is based on two successive steps: 1) the detection of abnormal events; and 2) the reaction against these abnormalities by specific algorithms. These two steps have been achieved by different methods. However, the principle is always to build an interactive device that reacts in a closed loop to the detected upper airway (UA) abnormality. Given the wide variety of algorithms used by these devices [15-18], clinical tests do not allow general conclusions to be derived for particular apparatus that are valid in all patients.

In a recent review, BERRY et al. [19] emphasised the fact that no study has compared the technologies of APAP devices that may not give the same results in a given patient. A bench study has been proposed to evaluate the responses of automatic devices to well-defined breathing patterns of sleep apnoea-hypopnoea syndrome (SAHS) patients [20]. In this study, the devices were evaluated in an open loop state, i.e. with a steady imposed perturbation of airflow, which remained unchanged by the automatic response of the APAP. In other words, steady patterns of disturbed breathing, stored in a computer, were applied to APAP devices, which could react by changing pressure. Nevertheless, airflow remained unaffected by these pressure changes, a condition that is unrealistic compared to clinical situations. Indeed, any change in airway pressure is accompanied by a change in UA size. Consequently, airflow pattern is modified. Therefore, this method could only challenge the event detection system, but could not test the pressure-response algorithms of the devices in reaction to secondary change in airflow pattern.

Several studies have shown that the mechanical properties of UA in children and adults with OSAS can be predicted by the behaviour of a collapsible tube described as a Starling resistor [21-24]. Therefore, this model was used to study the response of APAP devices to mechanical behaviour of the UA.

The current authors aimed to define the detection ability of the APAP and their potential capacity to correct breathing abnormalities during reproducible UA perturbation. Therefore, a model of the respiratory system was proposed, which allowed evaluation of the closed-loop behaviour of the APAP, as a first step in determining the effectiveness of each apparatus in a therapeutic context. 


\section{Materials and methods}

\section{Sinusoidal pump}

A custom-made pump (fig. 1) generated a sinusoidal airflow, at a rate of 12 cycles $\cdot \mathrm{min}^{-1}$, and a tidal volume (TV) of $1 \mathrm{~L}$. It was connected to a Starling resistor by an extendable tube acting as the trachea.

\section{Starling resistor}

The Starling resistor was made from a cylindrical airtight transparent chamber $(220 \mathrm{~mm}$ long, $90 \mathrm{~mm}$ internal diameter) containing a compliant rubber tube, as described by FARRÉ et al. [25].

The "larynx" was connected to the tracheal tube, whilst the "nose" was connected to the patient circuit (fig. 1). Pm and tracheal pressure $(P \operatorname{tr})$ were measured (Validyne DP45-16, $\pm 50 \mathrm{cmH}_{2} \mathrm{O}$ and DP45-24, $\pm 50 \mathrm{cmH}_{2} \mathrm{O}$; Validyne, Northridge, CA, USA). $P \operatorname{tr}$ was used as an index of the respiratory efforts to indicate the severity of the UA collapse. The chamber pressure $(P$ tis $)$ was controlled by a CPAP (Somnotron3; Weinmann, Hamburg, Germany) and a continuous negative airway pressure (CNAP), derived from a modified positive pressure device (Remstar; Respironics Inc, Murrysville, PA, USA). The two devices were mounted in parallel, using a T-piece adapted on the sealed box. The box pressure was monitored by a calibrated digital manometer (Respironics Inc). Normal breathing was simulated when the CNAP was powered on. The negative pressure acted as an increase in the UA dilator muscle activity. Partial or total collapse was simulated when the CPAP was powered on. The positive pressure around the collapsible tube acted as a decrease in the UA dilator muscle force.

The rubber tube critical closing pressure was evaluated at the mask by replacing the APAP by the CNAP, to apply negative pressure at the "nose". $P$ tis was then set at $0 \mathrm{cmH}_{2} \mathrm{O}$ in the box, and $P \mathrm{~m}$ was progressively decreased [24]. A closing pressure of $-2.2 \mathrm{cmH}_{2} \mathrm{O}$ was observed at complete interruption of inspiratory airflow.

\section{Patient circuit}

Each APAP system was connected, using its proprietary tube, to a conventional leak port ( $3 \mathrm{~mm}$ diameter) and to a pneumotachometer (Hans Rudolph 4700 Series; Hans Rudolph Inc, Kansas City, MO, USA). The leak port is used to avoid $\mathrm{CO}_{2}$ rebreathing in patients. Airflow was measured by using a differential transducer (Validyne DP4516, $\pm 2 \mathrm{cmH}_{2} \mathrm{O} ;$ Validyne). The pneumotachometer was connected to the Starling resistor using a rigid tube containing a manual tap to simulate "mask" leaks.

\section{Study devices}

The study included five APAP systems commonly used for treatment of sleep breathing disorders: GK 418P (Mallinckrodt, Villers Les Nancy, France), Autoset $\mathbb{R} \mathrm{T}$ (ResMed, North Ryde, Australia), REMstarAuto $\mathbb{R}$ (Respironics), PV10i (Breas Medical, Mölnlycke, Sweden) and

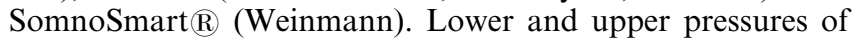
the devices were set at 4 and $18 \mathrm{cmH}_{2} \mathrm{O}$, respectively, whilst the pressure ramp was disabled. On the GK 418P, the maximum pressure in response to apnoea was set at $13 \mathrm{cmH}_{2} \mathrm{O}$. This device was set, initially, to react in the presence of central apnoeas and, secondly, to ignore them. The pressure rate was set on the SomnoSmart $\mathbb{R}$ to $0.4 \mathrm{cmH}_{2} \mathrm{O} \cdot \mathrm{s}^{-1}$. The other devices were used in their default setting given by the manufacturers.

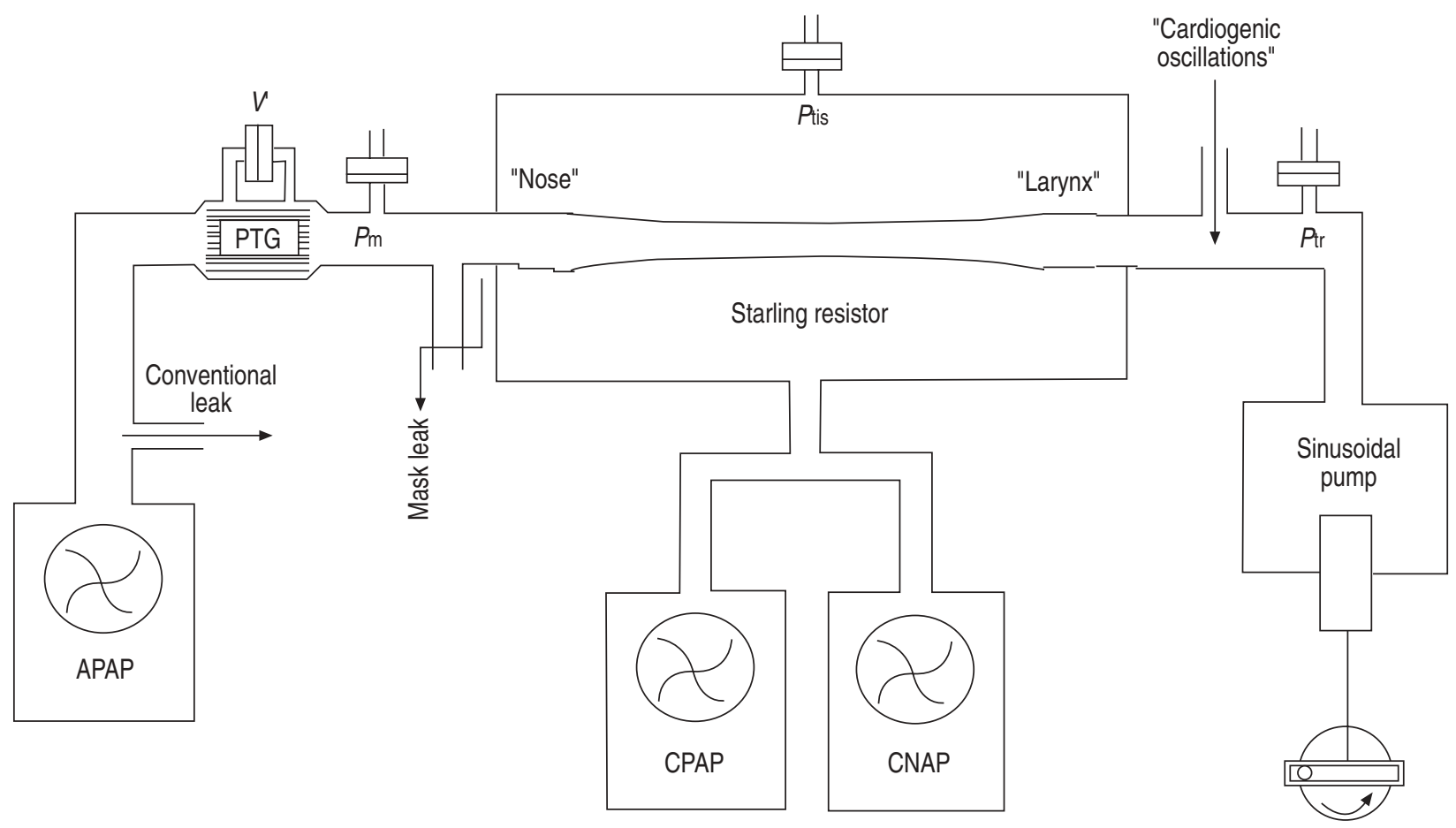

Fig. 1. - Experimental set-up used to submit auto-adjusting positive airway pressure (APAP) to different airflow patterns. $V^{\prime}$ : measured airflow; PTG: pneumotachograph; $P$ m: measured mask pressure; $P$ tis: measured tissular pressure; $P$ tr: measured tracheal pressure; CNAP: continuous negative airway pressure; CPAP: continuous positive airway pressure. 


\section{Signal acquisition}

Signals were sampled at a frequency of $50 \mathrm{~Hz}$ and collected using a 12-bit Analog to Digital converter (MacADIOS II; GW Instruments Inc, Sommerville, MA, USA). Airflow was calibrated using a rotameter and continuous flow source. The $\mathrm{TV}$ of each cycle was computed after digital integration of the measured airflow.

\section{Protocol}

Different flow patterns (fig. 2) were generated by the mechanical model. Airflow through the collapsible tube was managed by the relationship between $P \mathrm{~m}, P \operatorname{tr}$ and $P$ tis. Briefly, if $P \mathrm{~m}>P \operatorname{tr}>P$ tis, then the airflow pattern is normally rounded. If $P \mathrm{~m}>P$ tis $>P \operatorname{tr}$, the model snores and the airflow is flattened. Finally, if $P \operatorname{tr}<P \mathrm{~m}<P$ tis, the airway conduct becomes obstructed [26].
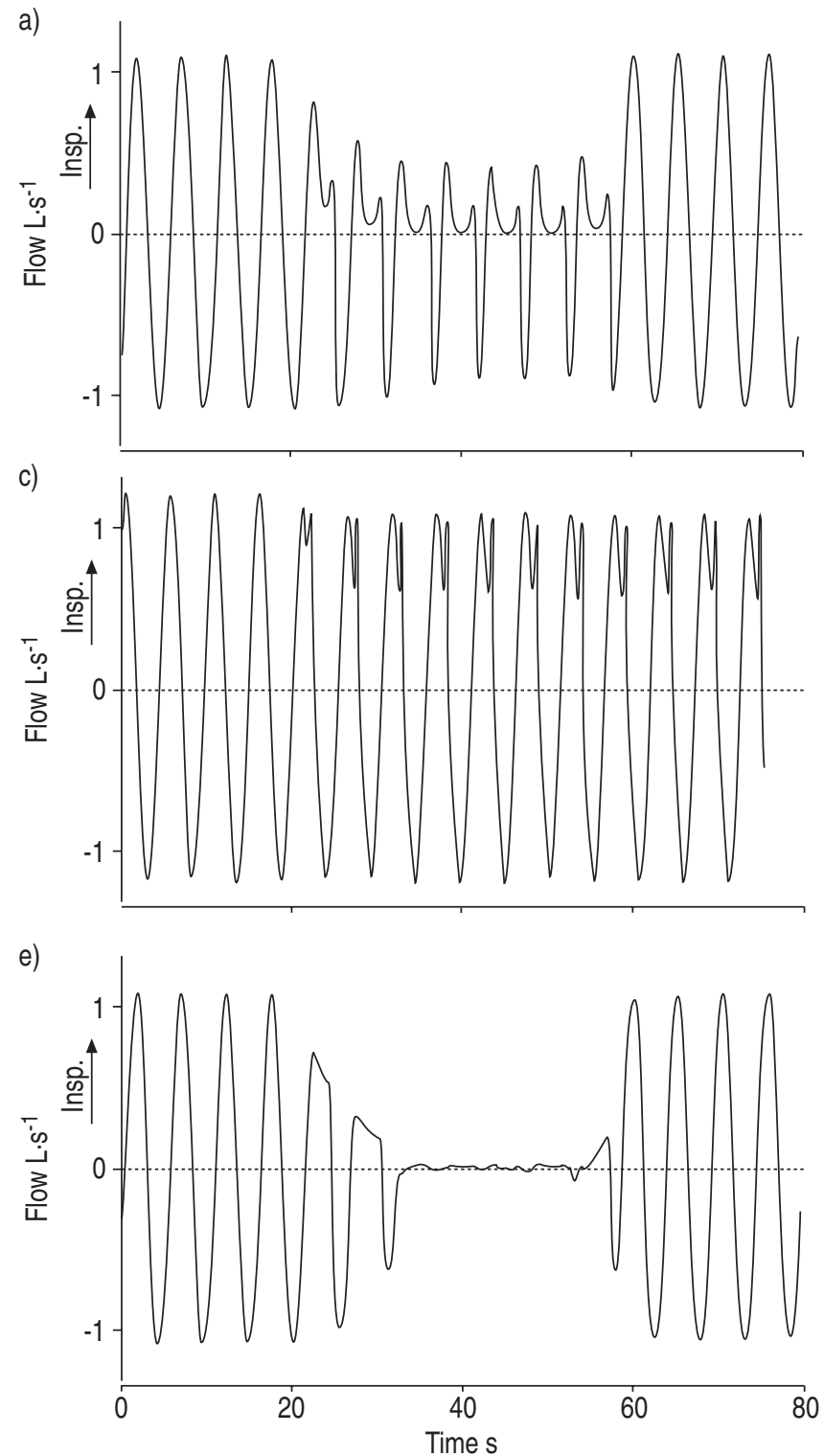

Normal airflow shape. The pharyngeal tube presented a flat cross-section that collapsed easily and had virtually no crosssectional area at zero transmural pressure. It was then necessary to apply negative pressure inside the box to maintain its open state and, thus, to obtain a rounded airflow shape. A cycle was considered as normal when the TV was $\geqslant 95 \%$ of baseline volume.

Hypopnoea. Hypopnoea was defined when $10 \%<\mathrm{TV}<70 \%$. U-shaped and square-shaped repetitive hypopnoeas were generated separately. A flattened inspiratory U-shaped pattern was characterised by two rapid inspiratory flow peaks in the same cycle, and snoring was often present. The first peak was present at the beginning of the inspiration and the second one at the end. To obtain a flattened square shape, a balloon of 3 L (Datex-Ohmeda, Helsinki, Finland) was placed in parallel with the sinusoidal pump. This compliant element was added to damp the inspiratory flow peaks observed with the U-shape and to suppress the snoring noise.
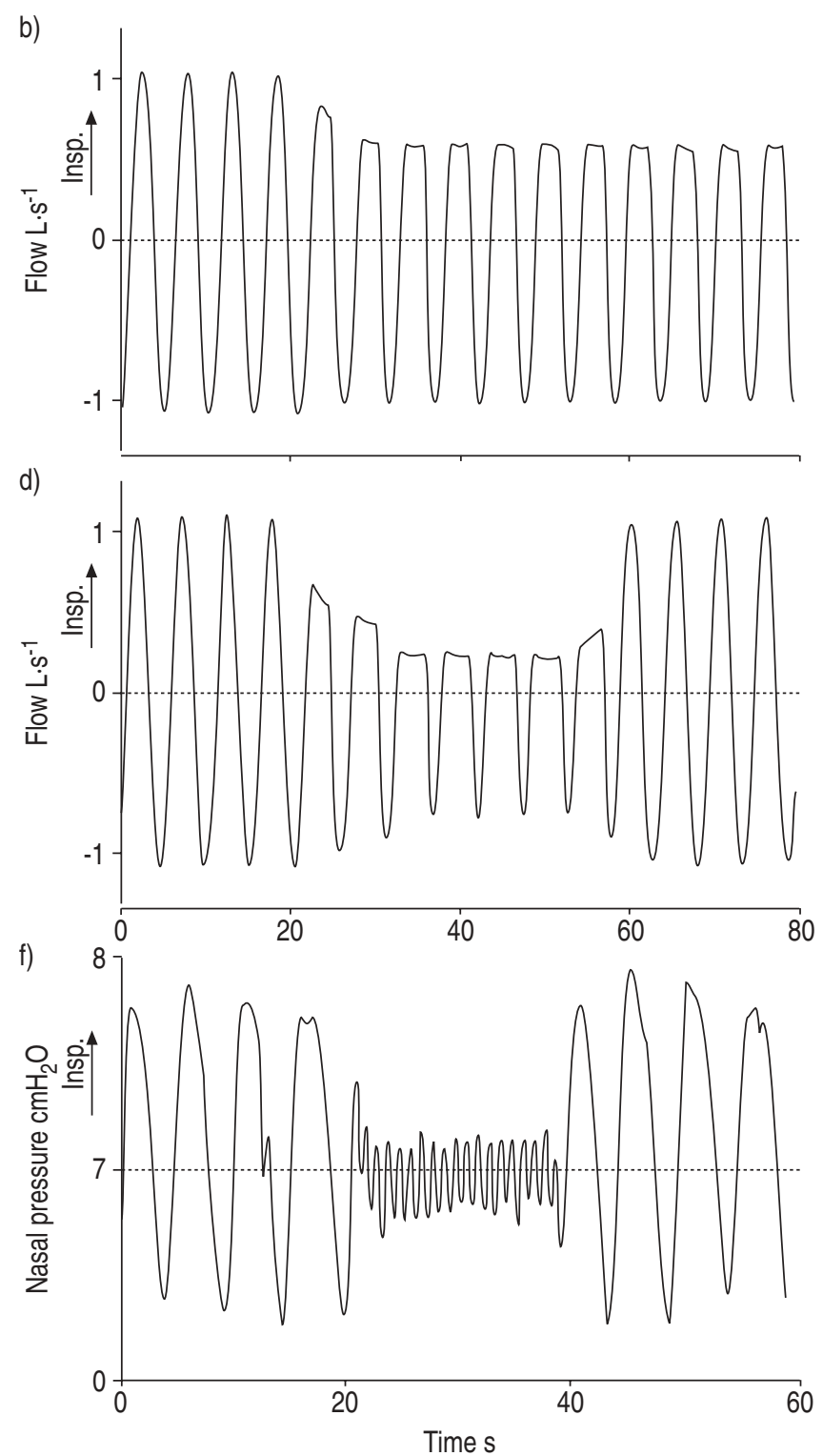

Fig. 2.-Patterns generated by the mechanical model are as follows: a) U-shaped hypopnoeas situated between two normal breathing periods; b) long period of flow limitation beginning at $20 \mathrm{~s}$; c) persistent snoring period beginning at 20 s; d) square-shaped hypopnoea situated between two normal breathing periods; e) a period of obstructive apnoea; and f) a period of central apnoea with visible pressure oscillations between 20 and $40 \mathrm{~s}$. Insp.: inspiration. 
Obstructive apnoea. When the tube was obstructed, low negative values were reached by $P \operatorname{tr}\left(-50 \mathrm{cmH}_{2} \mathrm{O}\right)$ as a result of the pump movements, which were similar to the efforts observed in human obstructive apnoeas. The TV decreased to values $\leqslant 10 \%$ baseline.

Flow limitation. Flow limitation was defined as $70 \% \leqslant \mathrm{TV}<95 \%$. As a balloon was used, as explained previously, no snoring was observed on both pressure and flow signals.

Simulated snoring. Snoring resulted from the flutter of the collapsible tube. Snoring was audible and was not associated with significant airflow reduction. The fluttering was visible on the rubber tube at its connection with the larynx. The snoring fundamental frequency was $\sim 105 \mathrm{~Hz}$.

Central apnoeas. Repetitive central apnoeas with opened UA were produced whilst the pump was shut down. In the meantime, an oscillation at $1 \mathrm{~Hz}$ was applied at the mask, by an external source, to simulate cardiogenic flow oscillations. The external source consisted of a rodent respiratory pump (UGO BASILE, Comerio, Italy). The displaced volume was $0.1 \mathrm{~mL}$ and the resultant pressure oscillation was $\sim \pm 0.5 \mathrm{cmH}_{2} \mathrm{O}$.

Mask leaks. APAP systems were submitted to a continuous controlled mask leak of $0.8 \mathrm{~L} \cdot \mathrm{s}^{-1}$ in the normal breathing state.

Events were manually created at a rate of $\sim 70-77 \cdot \mathrm{h}^{-1}$. Between each experiment, the APAP systems were allowed to return to their minimal pressure value. Thus, each airflow pattern was simulated, whilst the APAP was initially set at $4 \mathrm{cmH}_{2} \mathrm{O}$.

The time taken to reach maximal APAP in response to an event was defined as the reaction time $(t \mathrm{R})$. To evaluate the effectiveness of the response, the residual apnoea and hypopnoea indices (AI and HI, respectively) were calculated once the device reached its maximum pressure.

\section{Results}

\section{Response to apnoeas}

Figure 3 shows partial recordings of 15 min when obstructive apnoeas were imposed. The first part of each plot shows the initial $12.5 \mathrm{~min}$ of the recording, whereas the second part represents the final pressure reached by the different devices and the corresponding airflow during $2.5 \mathrm{~min}$. The

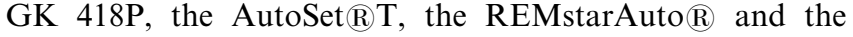
SomnoSmart $\mathbb{R}$ progressively changed apnoeas to hypopnoeas, whereas the PV10i completely suppressed the obstruc-

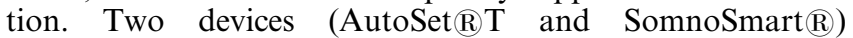
increased the pressure by large increments, whereas the others increased the pressure more smoothly. The complete recording is summarised in figure 4 and table 1.

Two devices (AutoSet $\mathbb{R} \mathrm{T}$ and GK 418P) suppressed apnoeas with a relatively short $t \mathrm{R}$, but maintained U-shaped hypopnoeas with a high residual index for the AutoSet $\mathbb{R} T$ (HI $55 \cdot \mathrm{h}^{-1}$ ). Whilst the other devices abolished apnoeas and hypopnoeas, they maintained flow-limited cycles. The maximum pressures reached ranged from $11.5 \mathrm{cmH}_{2} \mathrm{O}$ (AutoSet $\mathrm{R}$ ) to $16 \mathrm{cmH}_{2} \mathrm{O}$ (SomnoSmart $\AA$ ).

\section{Response to hypopnoeas}

When simulating hypopnoeas with a U-shape (fig. 5, table 2), maximum pressure values varied between $5.5 \mathrm{cmH}_{2} \mathrm{O}$
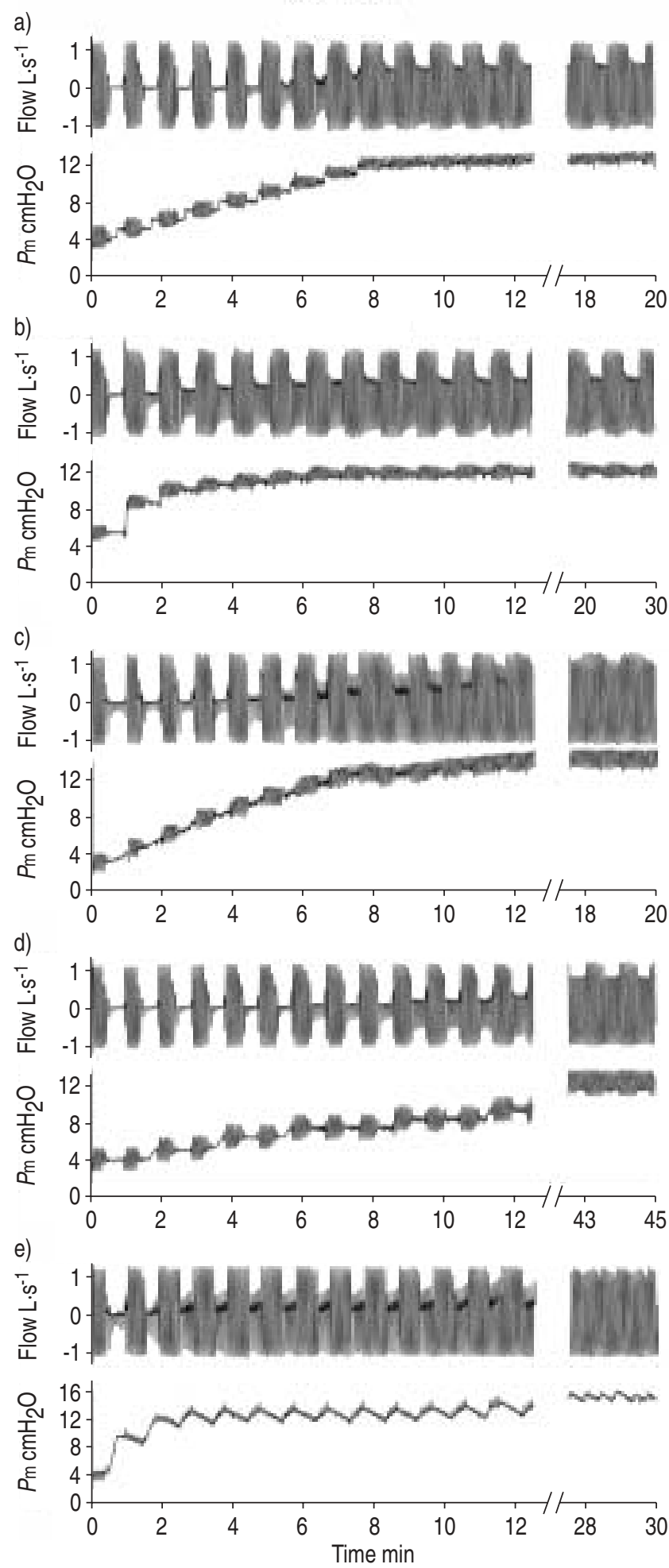

Fig. 3.-Automatic reaction from the auto-adjusting positive airway pressure (APAP) system when submitted to repetitive obstructive apnoeas. For each APAP system (a) GK 418P, b) Autoset $\mathbb{R}$ T, c) PV10i, d) REMstarAutoß, e) SomnoSmart $(\mathbb{R})$, the measured airflow and the mask pressure $(P \mathrm{~m})$ during a period of 15 min are plotted.

(AutoSet $\mathbb{R}$ T) and $9 \mathrm{cmH}_{2} \mathrm{O}$ (SomnoSmart $\mathbb{R}$ ). Whilst the GK $418 \mathrm{P}$ did not react to this event, the AutoSet $\mathbb{R} \mathrm{T}$ increased the pressure at the initial flattened cycles, but not enough to overcome the hypopnoea. With the REMstarAuto $\mathbb{R}$ and the PV10i, $P \mathrm{~m}$ values were $6.2 \mathrm{cmH}_{2} \mathrm{O}$ and $6.8 \mathrm{cmH}_{2} \mathrm{O}$, 


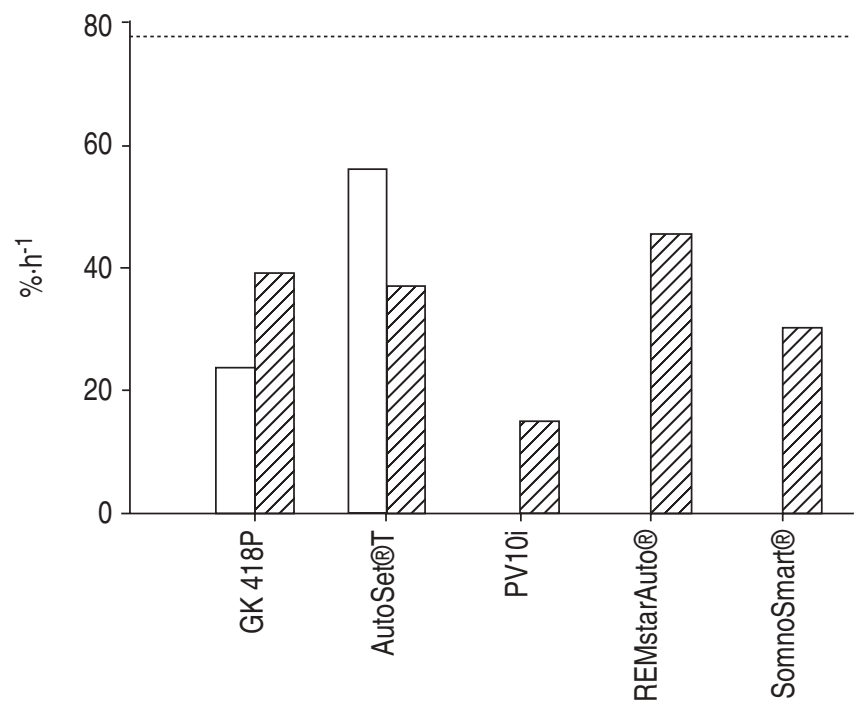

Fig. 4. - Residual hypopnoea indices $\left(\cdot \mathrm{h}^{-1} ; \square\right)$ and percentage of flowlimited cycles $(\%$; $\mathbb{Z})$ computed when continuous positive airway pressures were submitted to periodic obstructive apnoeas and once the maximum mask pressure $\left(P_{\max }\right)$ was reached. $P_{\max }$ was achieved at different reaction times for each device (table 1$)$. The dashed horizontal line represents the imposed apnoea index.

respectively. Consequently, all hypopnoeas were suppressed.

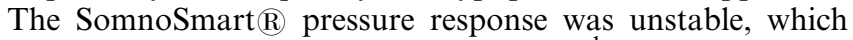
explains the low residual index $\left(\mathrm{HI} 9 \cdot \mathrm{h}^{-1}\right)$ despite its high pressure $\left(P \mathrm{~m} 8.7 \mathrm{cmH}_{2} \mathrm{O}\right)$. $t \mathrm{R}$ values were shorter than in the case of obstructive apnoeas $(2-6 \mathrm{~min})$. The AutoSet $\AA \mathrm{T}$, the PV10i and the REMstarAuto $\mathbb{R}$ suppressed all hypopnoeas with a square shape (fig. 6), whereas the GK 418P decreased the index to $27 \cdot \mathrm{h}^{-1}$. The SomnoSmart $\AA$ did not reduce this index below 59. As for $t \mathrm{R}$ values, they differed from that of the U-shaped hypopnoeas (1-10 $\mathrm{min})$.

The results presented in table 3 show the differences in residual hypopnoea indices when event classification was based on tidal volume (HITV), maximal inspiratory airflow (HIF1) and the difference between minimal to maximal flow, computed cycle by cycle (HIF2). For all methods, the same limits in percentage were used to identify events as described in the Protocol section. Only the simulated obstructive apnoeas and hypopnoeas were considered. For the squareshaped hypopnoeas and obstructive apnoeas, flow-derived residual indices (HIF1 and HIF2) were higher than volumederived indices (HITV) on the GK 418P. On the REMstarAuto $\AA$, HIF1 was higher when considering square-shaped hypopnoeas and obstructive apnoeas.

With the PV10i, residual square-shaped hypopnoeas persisted when computed by HIF2.

Finally, all devices maintained a significant number of flattened cycles (figs 4, 5 and 6).

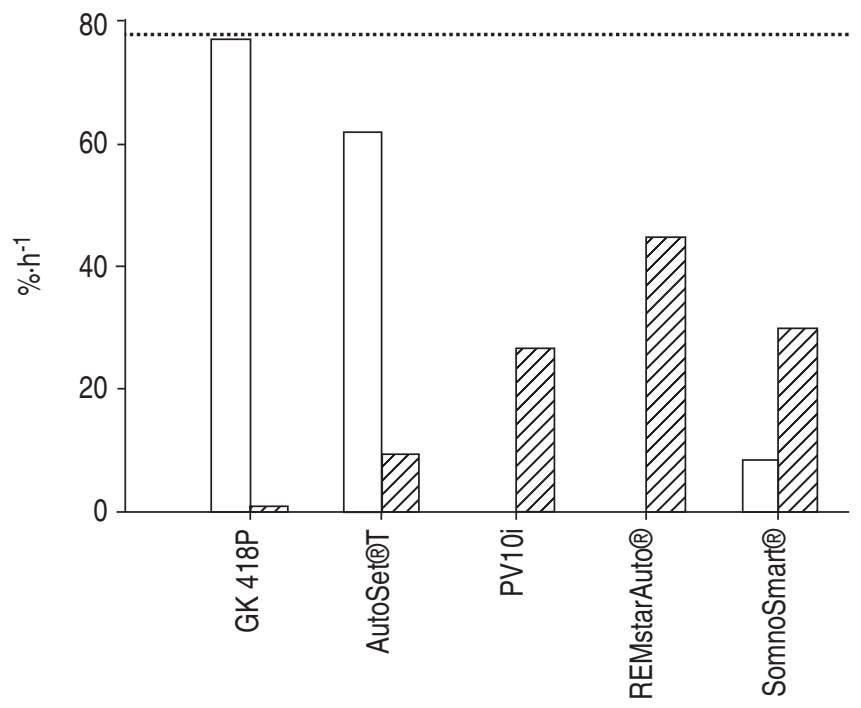

Fig. 5. - Residual hypopnoea indices $\left(\cdot \mathrm{h}^{-1} ; \square\right)$ and percentage of flowlimited cycles $(\%$; $\mathbb{Z})$ computed when continuous positive airway pressures (CPAP) were submitted to periodic U-shaped hypopnoeas. Corresponding reaction time and maximum measured mask pressure are given for each automatic CPAP in table 2. The dashed horizontal line represents the imposed apnoea index.

\section{Response to flow limitation and snoring}

Persistent flow limitations were not suppressed, even if some devices (AutoSet $\AA$ T, SomnoSmart $\AA$ and GK 418P) increased the pressure at the first flattened cycles and maintained this level thereafter. In spite of this increased pressure, $100 \%$ residual flow-limited cycles were observed.

Simulated snoring was detected and corrected by all devices except the AutoSet $\mathbb{R}$ T. The other devices raised the pressure

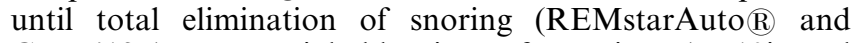
GK 418P) or partial blunting of snoring (PV10i and SomnoSmart $\AA$ ).

\section{Response to central apnoeas}

All the devices, except the SomnoSmart $\AA$, increased the pressure with central apnoeas. The AutoSet $\AA \mathrm{T}$ and the PV10i increased and maintained the pressure at 10 and $11 \mathrm{cmH}_{2} \mathrm{O}$, respectively, whereas the REMstarAuto $\AA$ increased the pressure to $11 \mathrm{cmH}_{2} \mathrm{O}$, before decreasing it to $8 \mathrm{cmH}_{2} \mathrm{O}$. The GK $418 \mathrm{P}$ was initially programmed not to react on central apnoeas with cardiac oscillations. Nevertheless, the pressure increased to $9.5 \mathrm{cmH}_{2} \mathrm{O}$. When the GK $418 \mathrm{P}$ was set to react to these events, the pressure further rose to $13 \mathrm{cmH}_{2} \mathrm{O}$.

Table 1.-Maximum mask pressure $\left(P_{\max }\right)$ at different reaction times $(t R)$ for each auto-adjusting positive airway pressure (APAP) device, when continuous positive airway pressures were submitted to periodic obstructive apnoeas

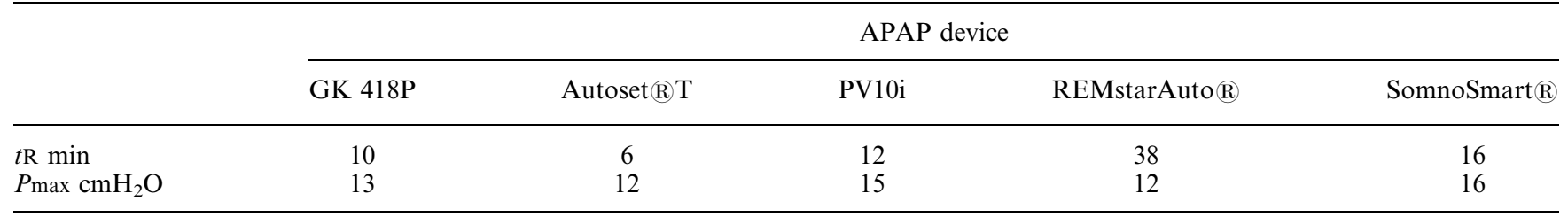

Data are presented as $n$. 
Table 2. - Maximum mask pressure $\left(P_{\max }\right)$ at different reaction times $(\mathrm{tR})$ for each auto-adjusting positive airway pressure (APAP) device, when continuous positive airway pressures were submitted to periodic U-shaped hypopnoeas

\begin{tabular}{|c|c|c|c|c|c|}
\hline & \multicolumn{5}{|c|}{ APAP device } \\
\hline & GK 418P & Autoset $\mathbb{R} \mathrm{T}$ & PV10i & REMstarAuto® & SomnoSmart $\mathbb{R}$ \\
\hline$t \mathrm{R} \min$ & $\infty^{\#}$ & 6 & 3 & 4 & 2 \\
\hline$P \max \mathrm{cmH}_{2} \mathrm{O}$ & 4 & 6 & 7 & 6 & 9 \\
\hline
\end{tabular}

Data are presented as n. ${ }^{\#}$ : no reaction was observed.

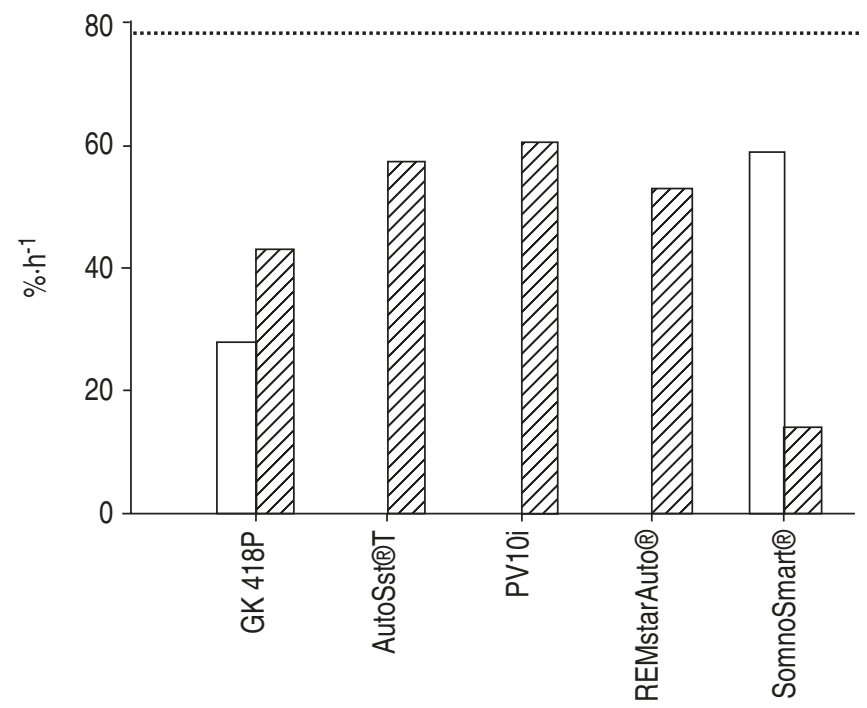

Fig. 6. - Residual hypopnoea indices $\left(\cdot \mathrm{h}^{-1} ; \square\right)$ and percentage of flowlimited cycles $(\% ; \mathbb{Z})$ computed when auto-adjusting positive airway pressure devices were submitted to periodic square-shaped hypopnoeas. The dashed horizontal line represents the imposed apnoea index. Corresponding reaction time and maximum $P_{\mathrm{m}}$ are given in table 4.

\section{Response to constant mask leaks}

The imposed leak resulted in a $P$ m drop and airflow drift (fig. 7). Consequently, inspiratory airflow became abnormally flat and was associated with high inspiratory efforts $(P \operatorname{tr}$ $-30 \mathrm{cmH}_{2} \mathrm{O}$ ), indicating a flow limitation. The device reacted to this event, and the airflow shape normalised again once the $P \mathrm{~m}$ returned to pre-leak value. This pattern was observed

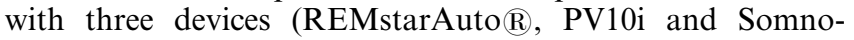

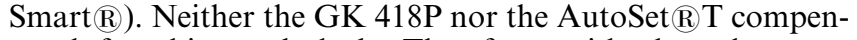
sated for this mask leak. Therefore, with these last two devices, the airflow pattern remained abnormal.

\section{Relationship between airflow and tracheal pressure}

The airflow shape is closely associated with the tracheal pressure. This is illustrated in figure 8 , where an improvement of airflow shape is accompanied by an increase of both airflow amplitude and tidal volume and a reduction of $P \operatorname{tr}$ swings.

\section{Discussion}

The main results of this study are as follows. 1) Tested APAPs are able to detect and to overcome obstructive

Table 3. - Observed residual hypopnoea indices (events $\left.\cdot \mathrm{h}^{-1}\right)$ using the tidal volume (HITV), maximal inspiratory airflow (HIF1) and the difference between minimal to maximal flow (HIF2)

\begin{tabular}{|c|c|c|c|c|c|c|c|c|c|}
\hline & \multicolumn{3}{|c|}{ U-shaped hypopnoea } & \multicolumn{3}{|c|}{ Square-shaped hypopnoea } & \multicolumn{3}{|c|}{ Obstructive apnoea } \\
\hline & HITV & HIF1 & HIF2 & HITV & HIF1 & HIF2 & HITV & HIF1 & HIF2 \\
\hline GK 418P & 77 & 77 & 77 & 28 & 60 & 60 & 24 & 59 & 47 \\
\hline Autoset $\mathbb{R} \mathrm{T}$ & 62 & 61 & 61 & 0 & 0 & 0 & 56 & 56 & 56 \\
\hline PV10i & 0 & 0 & 0 & 0 & 0 & 20 & 0 & 0 & 0 \\
\hline REMstarAutoß & 0 & 6 & 0 & 0 & 63 & 0 & 0 & 66 & 0 \\
\hline SomnoSmart $\AA$ & 9 & 13 & 0 & 59 & 63 & 59 & 0 & 0 & 0 \\
\hline
\end{tabular}

Data are presented as $n$.

Table 4.- Maximum mask pressure $\left(P_{\max }\right)$ at different reaction times $(\mathrm{t})$ for each auto-adjusting positive airway pressure (APAP) device submitted to periodic square-shaped hypopnoeas

$$
\text { APAP device }
$$

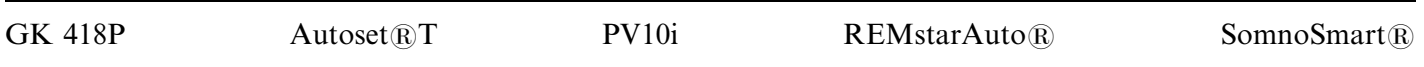

\begin{tabular}{llllll}
\hline$t \mathrm{R} \min$ & 2 & 7 & 5 & 10 & 1 \\
$P \max \mathrm{cmH}_{2} \mathrm{O}$ & 6 & 7 & 7 & 7 & 5
\end{tabular}

Data are presented as $\mathrm{n}$. 

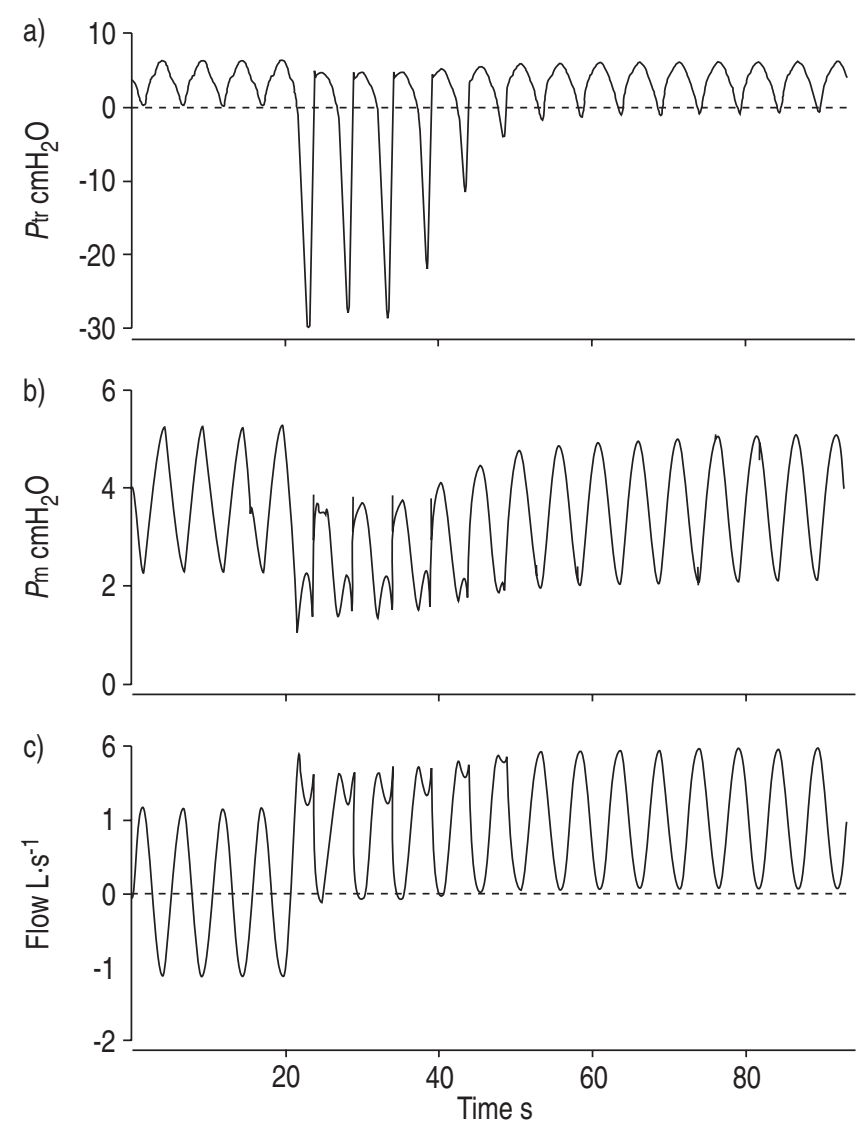

Fig. 7. - An example of a mask leak inducing a reaction of the autoadjusting positive airway pressure device. A leak was created at $\mathrm{t}=20 \mathrm{~s}$, inducing an abrupt increase of the tracheal pressure $(P \mathrm{tr})$ swings (a), a mask pressure $(P \mathrm{~m})$ drop (b), and an upward shift and a flattening of the airflow (c). At $\sim \mathrm{t}=50 \mathrm{~s}$, the device compensated for the leak, and the $P_{\mathrm{m}}$ increased back to the initial level. The tracheal pressure swings normalised and the airflow shape became rounded again at $\sim \mathrm{t}=60 \mathrm{~s}$.
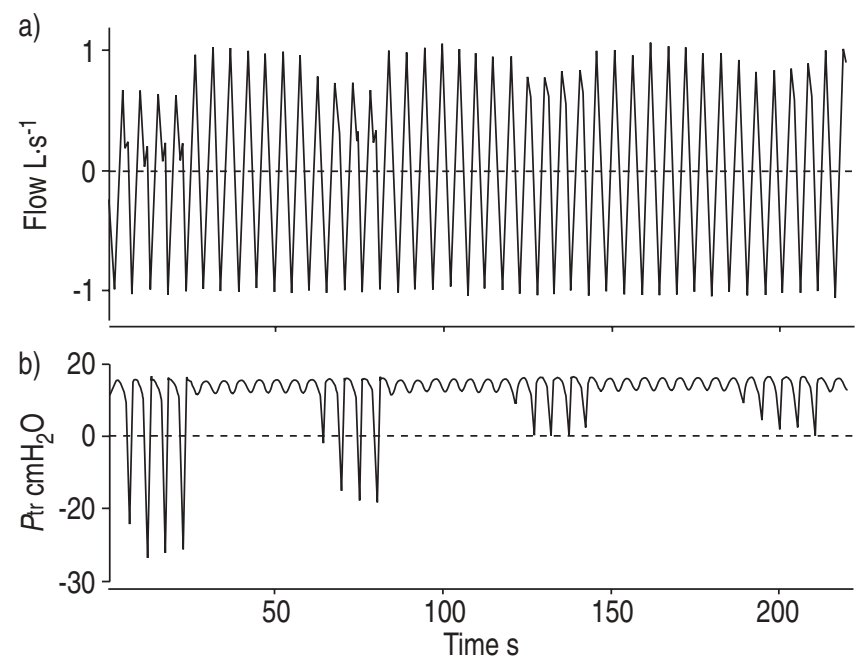

Fig. 8. - Four repetitive flow-limitation periods separated by normal flow shape. Tracheal pressure $(P \mathrm{tr} ; \mathrm{b})$ showed periodic high swings accompanied by airflow decreases (a). The corresponding minimum values of tidal volume during these periods of flow limitation were as follows: $77 \%(0-50 \mathrm{~s}) ; 85 \%(50-100 \mathrm{~s}) ; 92 \%(100-150 \mathrm{~s})$; and $93 \%$ $(150-200 \mathrm{~s})$. apnoeas, but, nevertheless, all devices maintained significant residual hypopnoeas and flow limitations (GK 418P and

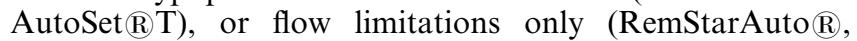
PV10i and SomnoSmart ( ). 2) One device did not fully normalise the hypopnoeas in either shape (GK 418P). The AutoSet $\mathbb{R} \mathrm{T}$ did not correct the U-shaped hypopnoeas,

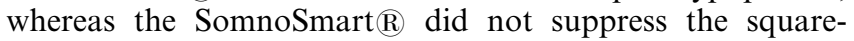
shaped ones. The other two devices corrected hypopnoeas, but left significant flow limitations. 3) Persistent flow limitation was not modified by any device, despite small pressure

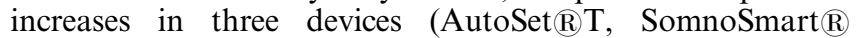
and GK 418P). 4) Snoring was suppressed with various $t \mathrm{R}$ by all devices, except for the AutoSet $\mathrm{R}$ T. 5) Mask leaks of $\approx 0.8 \mathrm{~L} \cdot \mathrm{s}^{-1}$ were not compensated for in two devices (AutoSet $\mathbb{R}$ T and GK 418P), even though the reduction in $P$ m caused an abnormal airflow. 6) The SomnoSmart $\mathbb{R}$ was the only device to maintain a low pressure at $4 \mathrm{cmH}_{2} \mathrm{O}$ when central apnoeas were simulated.

As well as UA anatomy, muscle activity plays a great role in the amount of air that can flow through the pharynx. Using the Starling resistor model, the mechanical effect of the UA muscle activity on the pharynx could be taken into account. Thus, allowing the APAP device to react on the model of the UA led to a more realistic approach of the overall functioning of the devices than in a previous bench study [20]. FARRÉ et al. [20] used airflow patterns from recorded patients and submitted APAP devices to these patterns. However, this study did not allow the APAPs to modify the airflow pattern despite their pressure increase, since the abnormal airflow sequences were programmed and imposed. Such a situation is unrealistic, since this open loop model cannot provoke the expected UA modifications that result from the APAP reaction. Only a closed loop method can do this. Thus, the major difference between the two models is that the current authors' closed loop setup reacted not only to pressure changes imposed around the collapsible tube, i.e. Ptis, but also to those generated by the APAP. This mechanical model simulated the situation of an active patient, whose breathing pattern depended on pressures applied both by the patient's UA muscles and by the APAP device. Thus, this model is better adapted to induce and evaluate APAP reaction to various patient situations. The airflow progressively changed from an abnormal shape to a normal shape as the APAP pressure increased. By contrast, in the FARRÉ et al. [20] model, obstructive events, such as apnoeas or hypopnoeas, remained imposed even at elevated $P \mathrm{~m}$ values, thereby preventing any evaluation of the improvement in flow patterns.

Obstructive events in patients may appear suddenly or be preceded by forerunner signs, such as snoring or flow limitation [27]. APAP devices' algorithms should probably detect these early signs to prevent total closure of the pharynx and to maintain the airway patency. Higher pressures are therefore required [28].

The snoring cycles were characterised by flattened shapes of both airflow and $P \mathrm{~m}$, in addition to an increase of the tracheal pressure swings. The fundamental oscillation frequency of snoring, in this model, was in the range of those observed in humans [29]. In a bench study, differences were reported between six commercially available APAP devices in their sensitivity for detecting snoring on a lung model [30]. The authors showed that snoring detection is less sensitive for the AutoSet $(\mathrm{R}$ T compared with the GK 418P. The complete lack of reaction to snoring of the AutoSet $\mathbb{R} T$ in the current authors' model may be related to the amplitude of the snoring pressure oscillation, which was $\sim 0.7 \mathrm{cmH}_{2} \mathrm{O}$, whereas in the LOFASO et al. [30] study, it was $\sim 1 \mathrm{cmH}_{2} \mathrm{O}$.

Flow-limited cycles were characterised by a decrease of TV, associated with large tracheal pressure swings (fig. 8). 
Prolonged flow limitations were not corrected and repetitive flow-limited cycles, separated by normal cycles, were not suppressed. However, response to prolonged flow-limitation events may not be desirable, as they are physiologically observed in stage 3-4 of sleep and they do not lead to repetitive arousals [31]. AitTOKALLIO et al. [32] emphasised the usefulness of advanced signal processing in classifying different airflow shapes. Furthermore, EPSTEIN et al. [33] showed that flattening, detected on a flow signal, provided by a nasal cannula, may be an alternative to invasive oesophageal pressure measurement. Even if a direct effort measurement is not readily available, APAP devices may have sufficient data to perform effective event recognition, by evaluating the severity of the flattening using flow-shape analysis.

In the current study, the criteria used to define hypopnoea and apnoea events are based on precise quantitative measurements of the actual TV of each cycle. The American Academy of Sleep Medicine criterion [34] is a decrease of $\geqslant 50 \%$ of baseline amplitude of a valid measurement of breathing during sleep. This method uses only peak-to-peak measurement of airflow, which is much less accurate than actual TV integrated over the total respiratory cycle. The current authors submitted APAPs to two realistic flattened patterns of hypopnoeas, commonly observed on SAHS patients [32]. The AutoSet $\mathrm{R} T$ did not suppress the Ushaped flattening and left an HI of $62 \cdot \mathrm{h}^{-1}$. This result was not expected with this device, since the algorithm is supposed to anticipate total airway closure. The pressure was raised by only $2 \mathrm{cmH}_{2} \mathrm{O}$, which suggests that the AutoSet $\mathrm{R} \mathrm{T}$ device recognised the abnormality, but did not increase the pressure sufficiently. The detection of U-shaped hypopnoeas also failed on the GK 418P, whereas the square shape was partially corrected. The PV10i and the REMstarAuto $\mathbb{R}$ eliminated all types of hypopnoeas, but maintained repetitive flow limitations. The SomnoSmart $\mathbb{R}$ increased the pressure in the flattened cycles and decreased it immediately in the normal ones. This latter device is driven by a simplified forced oscillatory technique, producing a $20 \mathrm{~Hz}$ oscillation and measuring an oscillating pressure signal (OPS). The OPS increases in the presence of a partial or total collapse of the UA. The $P \mathrm{~m}$ signal was consequently very unstable and fluctuating, resulting in the persistence of some U-shaped hypopnoeas (HI 9). With square-shaped hypopnoeas, the SomnoSmart $(\mathrm{R})$ did not increase the pressure $>5 \mathrm{cmH}_{2} \mathrm{O}$ and the HI remained high $\left(\mathrm{HI} 59 \cdot \mathrm{h}^{-1}\right)$. This low performance was probably due to the low values of OPS in this mode at inspiration compared with expiration. This may have resulted from the damping of the $20 \mathrm{~Hz}$ oscillations in the balloon.

All devices overcame obstructive apnoeas. The AutoSet $\mathbb{R} \mathrm{T}$

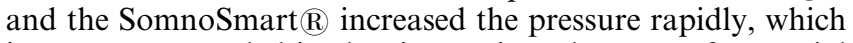
is not recommended in sleeping patients because of potential arousals [35].

The most frequently used signal to define events in the sleep laboratories is the airflow, where the peak flow amplitude and the inspiratory flow shape are used to identify events. By choosing the TV [20], the current authors used a more stringent criterion. To compare these methods, residual events were computed using volume and flow criteria (table 3). For the GK 418P and the REMstarAuto $\AA$, residual indices were higher when using the flow signal. These differences in residual indices are related to the fact that a cycle will be considered as only flow limited by analysing its volume, but it may be classified as a hypopnoea using its flow amplitude. The use of the flow signal instead of the TV may then lead to different residual indices. Thus, differences between APAP devices in reaction to respiratory events may result from different detection strategies. These results suggest that some devices did not suppress flattened cycles, as a result of their criteria for detecting abnormal flow.

However, the commonly used peak flow is not as representative of ventilation as the TV. As shown in figure 2, the U-shaped flow curve is characterised by a high peak flow at the beginning of the inspiration, whereas a reduction in mean flow amplitude is present during most part of the inspiration. Thus, this peak flow value does not necessarily reflect the severe decrease in amplitude that characterises this shape.

In the case of central apnoeas, pressure should not be increased [2, 36]. Nevertheless, all devices, except for the SomnoSmart $\AA$, increased the pressure. The forced oscillatory technique of the SomnoSmart $($ showed its sensitivity in differentiating between open and closed UA. The GK 418P monitoring software showed that these events were processed as obstructive or mixed apnoeas causing pressure increases. Only the PV10i and the AutoSet $\mathbb{R}$ T algorithms did not separate between obstructive and central apnoeas. These events were classified as apnoea. Even if the two latter devices are not specifically designed to treat central apnoeas, such events may occur in a given patient. In this case, an APAP device should provide a null or moderate pressure increase. The REMstarAuto $\mathbb{R}$ did not identify the central apnoeas with accuracy, but characterised them as nonresponsive events.

The current data show that mask leaks occurring at minimal effective positive pressure cause flow limitation or snoring associated with increased tracheal pressure swings. The hypothesis that APAP units monitoring flow or impedance may systematically fail to titrate properly because of high mask leaks [19] is disproven, as a result of the current data showing an adequate reaction by some devices (SomnoSmart $\mathbb{R}$, PV10i and REMstarAuto $\mathbb{R}$ ). The confusion between leak periods and apnoea or hypopnoea events [19] should not be possible when a proper flow-signal analysis is performed. A mask leak is systematically accompanied by opposite offsets of mask pressure and airflow signals (fig. 7).

The current authors' model has the advantage of wellcontrolled and stable conditions for APAP studies, so that each experiment is reproducible for each device. Such a comparison study is not possible on humans, as a result of intra- and inter-patient variability. Differences between patients in pharynx anatomy, AI/HI, snoring frequency, sleep quality, mask tolerance and noise susceptibility can be considered as explanatory factors of different outcomes between devices rather than actual device differences.

These results contrast with some positive reports of the clinical efficacy of APAP devices for automated CPAP titration [36, 37]. Reasons for these discrepancies may be related to event classification in other studies that take into account apnoeas and hypopnoeas, but not flow limitations. Another reason could be the clinical efficacy of some devices in snoring detection, which is often a forerunner sign of UA obstruction. Insofar as failures of detection algorithms with several devices have been detected, it is important to validate carefully event reports of APAP and to monitor their performance by independent means.

\section{Limitations of the study}

This model does not take into account all mechanisms of UA pathophysiology, such as variations in UA muscle activity within the respiratory cycle, the surface tension changes in UA or all possible airflow patterns. Despite these differences, the critical closing pressure of the model was close 
to values observed on normal subjects [38]. The current authors have tested only some specific airflow patterns similar to that observed in patients. Furthermore, the airflow waveforms were flattened at the inspiration only. Therefore, the current study could not cover all combinations of inspiratory and expiratory airflow abnormalities.

A mechanical model cannot evaluate directly if simulated hypopnoeas and flow-limited events precipitate desaturation and/or arousals, as these parameters are not built in to the model. However, there is strong indirect evidence that it should be the case in an equivalent clinical setting. Indeed, the high tracheal pressure decrements seen in figure 8 $\left(\sim 40 \mathrm{cmH}_{2} \mathrm{O}\right)$, during events labelled "hypopnoeas" and "flow-limited", are known to induce arousals in humans [39].

Furthermore, it is difficult to estimate actual desaturation from the model, since, besides hypoventilation, many factors are associated with sleep-disordered breathing, including variation in oxygen stores across patients, as well as potential variation in $\mathrm{O}_{2}$ consumption and $\mathrm{CO}_{2}$ production under sleep apnoeic/hypopnoeic/flow-limited conditions.

The capabilities of the devices to react against a mechanical model may be questioned when compared to better clinical results. However, elevated residual indices of hypopnoeas or flow limitation during simulated obstructive apnoeas were not related to a pressure limit of the devices, since all the machines did not reach the maximum pressure setting (maximum pressure was $16 \mathrm{cmH}_{2} \mathrm{O}$ with the SomnoSmart $\mathrm{R}$ and PV10i). This failure may be linked to a built-in limitation of the algorithms, which probably applies to clinical situations as well.

In conclusion, tested auto-adjusting positive airway pressure devices had different responses under the same experimental conditions. These differences are indicated by the amount of pressure increase and reaction time in response to the same event. Whilst all devices adequately detected and treated total upper airway collapse, some devices did not correct hypopnoeas. The detection of flow-limited cycles and differentiation between central and obstructive events needs to be improved in most devices, using accurate tools, such as signal processing. In addition, the current study suggests that it is necessary to visualise the raw data of the device to better evaluate its automatic response. This model of the upper airway is a valuable first step in comparing technological advances in the treatment of sleep apnoea-hypopnoea syndrome patients, but must be supplemented by clinical studies for devices that have proven their efficacy in the bench test.

\section{References}

1. Lloberes P, Ballester E, Montserrat JM, et al. Comparison of manual and automatic CPAP titration in patients with sleep apnoea/hypopnoea syndrome. Am J Respir Crit Care Med 1996; 154: 1755-1758.

2. Series F, Marc I. Efficacy of automatic continuous positive airway pressure therapy that uses an estimated required pressure in the treatment of the obstructive sleep apnoea syndrome. Ann Intern Med 1997; 127: 588-595.

3. Behbehani K, Yen FC, Lucas EA, Burk JR. A sleep laboratory evaluation of an automatic positive airway pressure system for treatment of obstructive sleep apnoea. Sleep 1998; 21: 485-491.

4. Loube DI. Technologic advances in the treatment of obstructive sleep apnoea syndrome. Chest 1999; 116: 14261433.

5. Series F. Is treatment of obstructive sleep apnoea syndrome with auto-CPAP useful? Sleep 2000; 23: S161-S165.

6. d'Ortho MP, Grillier-Lanoir V, Levy P, et al. Constant versus automatic continuous positive airway pressure therapy: home evaluation. Chest 2000; 118: 1010-1017.

7. Massie CA, McArdle N, Hart RW, et al. Comparison between automatic and fixed positive airway pressure therapy in the home. Am J Respir Crit Care Med 2003; 167: 20-23.

8. Meurice JC, Marc I, Series F. Efficacy of auto-CPAP in the treatment of obstructive sleep apnoea/hypopnoea syndrome. Am J Respir Crit Care Med 1996; 153: 794-798.

9. Series F. Accuracy of an unattended home CPAP titration in the treatment of obstructive sleep apnea. Am J Respir Crit Care Med 2000; 162: 94-97.

10. Stradling JR, Barbour C, Pitson DJ, Davies RJ. Automatic nasal continuous positive airway pressure titration in the laboratory: patient outcomes. Thorax 1997; 52: 72-75.

11. Restrick LJ, Fox NC, Braid G, Ward EM, Paul EA, Wedzicha JA. Comparison of nasal pressure support ventilation with nasal intermittent positive pressure ventilation in patients with nocturnal hypoventilation. Eur Respir $J$ 1993; 6: 364-370.

12. Piper AJ, Sullivan CE. Effects of short-term NIPPV in the treatment of patients with severe obstructive sleep apnoea and hypercapnia. Chest 1994; 105: 434 440.

13. Juhasz J, Schillen J, Urbigkeit A, Ploch T, Penzel T, Peter JH. Unattended continuous positive airway pressure titration. Clinical relevance and cardiorespiratory hazards of the method. Am J Respir Crit Care Med 1996; 154: 359-365.

14. Sharma S, Wali S, Pouliot Z, Peters M, Neufeld H, Kryger $M$. Treatment of obstructive sleep apnoea with a self-titrating continuous positive airway pressure (CPAP) system. Sleep 1996; 19: 497-501.

15. Teschler H, Berthon-Jones M. Intelligent CPAP systems: clinical experience. Thorax 1998; 53: S49-S54.

16. Shin JJ, Berry RB, Khoo MC. Fuzzy assessment of sleepdisordered breathing during continuous positive airway pressure therapy. Sleep 1998; 21: 817-828.

17. Randerath WJ, Schraeder O, Galetke W, Feldmeyer F, Rühle KH. Autoadjusting CPAP therapy based on impedance efficacy, compliance and acceptance. Am J Respir Crit Care Med 2001; 163: 652-657.

18. Farré R, Rotger M, Montserrat JM, Navajas D. A system to generate simultaneous forced oscillation and continuous positive airway pressure. Eur Respir J 1997; 10: 1349-1353.

19. Berry RB, Parish JM, Hartse KM. The use of auto-titrating continuous positive airway pressure for treatment of adult obstructive sleep apnoea. Sleep 2002; 25: 148-173.

20. Farré R, Montserrat JM, Rigau J, Trepat X, Pinto P, Navajas D. Response of automatic continuous positive airway pressure devices to different sleep breathing patterns: a bench study. Am J Respir Crit Care Med 2002; 166: 469473.

21. Schwartz AR, Smith PL, Wise RA, Gold AR, Permutt S. Induction of upper airway occlusion in sleeping individuals with subatmospheric nasal pressure. J Appl Physiol 1988; 64: 535-542.

22. Schwartz AR, Smith PL, Wise RA, Bankman I, Permutt S. Effect of positive nasal pressure on upper airway pressureflow relationships. J Appl Physiol 1989; 66: 1626-1634.

23. Smith PL, Wise RA, Gold AR, Schwartz AR, Permutt S. Upper airway pressure-flow relationships in obstructive sleep apnoea. J Appl Physiol 1988; 64: 789-795.

24. Marcus CL, McColley SA, Carroll JL, Loughlin GM, Smith PL, Schwartz AR. Upper airway collapsibility in children with obstructive sleep apnoea syndrome. J Appl Physiol 1994; 77: 918-924.

25. Farré R, Peslin R, Rotger M, Navajas D. Inspiratory dynamic obstruction detected by forced oscillation during CPAP. Am J Respir Crit Care Med 1997; 155: 952-956.

26. Gold AR, Schwartz AR. The pharyngeal critical pressure. The whys and hows of using nasal continuous positive airway pressure diagnostically. Chest 1996; 110: 1077-1088.

27. Ayappa I, Norman RG, Hosselet JJ, Gruenke RA, Walsleben JA, Rapoport DM. Relative occurrence of flow limitation and snoring during continuous positive airway pressure titration. Chest 1998; 114: 685-690. 
28. Montserrat JM, Ballester E, Olivi H, et al. Time-course of stepwise CPAP titration. Behavior of respiratory and neurological variables. Am J Respir Crit Care Med 1995; 152: $1854-1859$.

29. Liistro G, Stanescu D, Veriter C. Pattern of simulated snoring is different through mouth and nose. J Appl Physiol 1991; 70: 2736-2741.

30. Lofaso F, Leroux K, Quera-Salva MA, et al. Snoring detection during auto-nasal continuous positive airway pressure. Eur Respir J 2002; 19: 108-112.

31. Rees K, Kingshott RN, Wraith PK, Douglas NJ. Frequency and significance of increased upper airway resistance during sleep. Am J Respir Crit Care Med 2000; 162: 1210-1214.

32. Aittokallio T, Saaresranta T, Polo-Kantola P, Nevalainen O, Polo O. Analysis of inspiratory flow shapes in patients with partial upper-airway obstruction during sleep. Chest 2001; 119: $37-44$

33. Epstein MD, Chicoine SA, Hanumara RC. Detection of upper airway resistance syndrome using a nasal cannula/ pressure transducer. Chest 2000; 117: 1073-1077.

34. Sleep-related breathing disorders in adults: recommendations for syndrome definition and measurement techniques in clinical research. The Report of an American Academy of Sleep Medicine Task Force. Sleep 1999; 22: 667-689.

35. Fuchs FS, Wiest GH, Frank M, et al. Auto-CPAP therapy for obstructive sleep apnoea: induction of microarousals by automatic variations of CPAP pressure? Sleep 2002; 25: 514 518.

36. Teschler H, Berthon-Jones $\mathrm{M}$, Thompson AB, Henkel A, Henry J, Konietzko N. Automated continuous positive airway pressure titration for obstructive sleep apnea syndrome. Am J Respir Crit Care Med 1996; 154: 734 740.

37. Berthon-Jones M, Lawrence S, Sullivan CE, Grunstein R. Nasal continuous positive airway pressure treatment: current realities and future. Sleep 1996; 19: S131-S135.

38. Isono S, Remmers JE, Tanaka A, Sho Y, Sato J, Nishino T Anatomy of pharynx in patients with obstructive sleep apnoea and in normal subjects. J Appl Physiol 1997; 82: $1319-1326$.

39. Sforza E, Krieger J, Petiau C. Nocturnal evolution of respiratory effort in obstructive sleep apnoea syndrome: influence on arousal threshold. Eur Respir J 1998; 12: 12571263. 